FNAL-Pub-04/136-CD

\title{
Synergia: an Accelerator Modeling Tool with 3-D Space Charge
}

\author{
J. Amundson and P. Spentzouris \\ Fermi National Accelerator Laboratory, Batavia, IL 60510, USA \\ J. Qiang and R. Ryne \\ Lawrence Berkeley National Laboratory, Berkeley, California 94720, USA
}

(Dated: July 27, 2004)

\begin{abstract}
High precision modeling of space-charge effects, together with accurate treatment of singleparticle dynamics, is essential for designing future accelerators as well as optimizing the performance of existing machines. We describe Synergia, a high-fidelity parallel beam dynamics simulation package with fully three dimensional space-charge capabilities and a higher order optics implementation. We describe the computational techniques, the advanced human interface, and the parallel performance obtained using large numbers of macroparticles. We also perform code benchmarks comparing to semi-analytic results and other codes. Finally, we present initial results on particle tune spread, beam halo creation, and emittance growth in the Fermilab booster accelerator.
\end{abstract}

PACS numbers: 


\section{INTRODUCTION}

In recent years, accurate modeling of beam dynamics in high-current low energy proton synchrotrons has become necessary because of new machines under consideration for future applications, such as the High Energy Physics neutrino program, and the need to optimize the performance of currently operating machines, such as the Spallation Neutron Source or the Fermilab Booster. These machines are characterized by high currents and require excellent control of beam losses, thus space-charge initiated halo formation is an essential component of their modeling. In order to obtain accurate predictions for realistic conditions of operation, single-particle optics and self-consistent multi-particle effects must be combined in a single simulation code.

Several computer simulations of space-charge effects in circular accelerators using particlein-cell techniques have been developed [1-3]. These simulations have emphasized the transverse dynamics while using a less rigorous approach for the longitudinal dynamics. Synergia [4] is a package for state-of-the-art simulation of linear and circular accelerators with a fully three-dimensional treatment of space charge, and the ability to use arbitrary order maps for the single-particle optics modeling.

Synergia is designed to be a general-purpose tool with an interface that is accessible to accelerator physicists who are not experts in simulation. Space-charge calculations are computationally intensive, typically requiring the use of parallel computers. The implementation of Synergia is fully parallel, including the particle tracking and space-charge modules. The code itself is a hybrid system based on previously developed accelerator physics codes. Synergia includes enhancements to these codes as well as new integration and interface modules.

Development of Synergia has been funded by the United States Department of Energy's SciDAC Accelerator Science and Technology Project. One of the goals of the project is to create distributable codes. Since compiling hybrid code can be a complicated task which is further complicated by the diverse set of existing parallel computing environments, Synergia includes a build system that allows it to be compiled and run on various platforms without requiring the user to modify the code and/or build system.

In this paper we give a brief description of the components used in Synergia as well as the details involved in combining them into a single product. We pay close attention to the build system, in keeping with the "distributable" goal mentioned above. We also describe how we have taken advantage of Python to give us a flexible human user interface with very little effort. In addition, we present a few Synergia applications. First, we compare with analytic calculations and predictions from other codes to verify the accuracy of our implementation. Then, in order to demonstrate the capabilities of the code in a realistic scenario, we present results from simulations of the Fermilab Booster [5].

\section{COMPONENTS}

The two packages at the core of Synergia are IMPACT [7] and the mxyzptlk/beamline libraries [8]. We have added glue code and a human-interface wrapper to these packages, together with necessary extensions of their modules, to form the Synergia package. 


\section{A. IMPACT}

Synergia uses IMPACT for its parallel particle-in-cell (PIC) implementation, rf modeling and, most importantly, parallel space-charge calculations. IMPACT contains a suite of three-dimensional Poisson solvers that are invoked in the middle of each step of a splitoperator-based model. We split the Hamiltonian into two pieces,

$$
H=H_{\mathrm{ext}}+H_{\mathrm{sc}}
$$

where $H_{\text {ext }}$ is the Hamiltonian for the external beamline element part of the problem and $H_{\text {sc }}$ is the Hamiltonian for the space-charge part of the problem. In our case the latter is

simply proportional to the scalar potential, with a proportionality constant that varies as $\frac{1}{\gamma^{2}}$ to account for the azimuthal magnetic field associated with the longitudinal beam current. Since the scalar potential depends only on coordinates and not momenta, the effect of $H_{\mathrm{sc}}$ is a change in momentum, i.e. a space-charge kick, which we denote by $\mathcal{M}_{\mathrm{sc}}$. The effect of $H_{\text {ext }}$ is described by the transfer map for the associated beamline element, $\mathcal{M}_{\text {ext }}$. In the split-operator approach, an approximation to the effect of the full Hamiltonian, $H$, accurate through second order in the step size $h$, is given by

$$
\mathcal{M}(h)=\mathcal{M}_{\text {ext }}(h / 2) \mathcal{M}_{\text {sc }}(h) \mathcal{M}_{\text {ext }}(h / 2)+\mathcal{O}\left(h^{3}\right) .
$$

The problem of calculating beam propagation including space-charge effects therefore factorizes into the problem of calculating the two effects one at a time and combining them as above. A key advantage of this splitting, as opposed to one that separates the Hamiltonian into pieces involving only coordinates and only momenta, is that in our approach the rapid variation of the external fields is separated from the more slowly varying space-charge fields. In other words, we can take small steps to accurately resolve rf cavity fields, magnetic fringe fields, etc., needed to compute external transfer maps, but the separation between spacecharge kicks can be large (typically a few tens of kicks per betatron wavelength). Without the factorization above, we would be forced to calculate the space-charge effects on the time scale set by the magnetic optics effects, which would be computationally prohibitive.

We have extended the original IMPACT in several ways. IMPACT now includes an injection module, allowing multi-turn injection modeling. We have extended the beam generation module to include a six-dimensional Gaussian distribution with general correlations. (See Section III B 4.) We have also improved the memory management, allowing for an arbitrary number of beamline elements. Finally, we have enhanced the IMPACT particle data structure to allow following individual particles throughout the simulation and calculating particle tunes.

\section{B. mxyzptlk/beamline libraries}

The mxyzptlk/beamline package is a set of $\mathrm{C}++$ libraries covering a wide range of accelerator physics computations. Even though the original code is over 10 years old, the libraries are written in a modern style, including real objects with encapsulation and well-considered interfaces. The package includes basic_toolkit, a set of useful utility classes such as Vector, Matrix, etc., beamline, objects for modeling elements of a beamline, mxyzptlk, classes for automatic differentiation and differential algebra, and physics_toolkit, a set of classes for analysis and computation. 


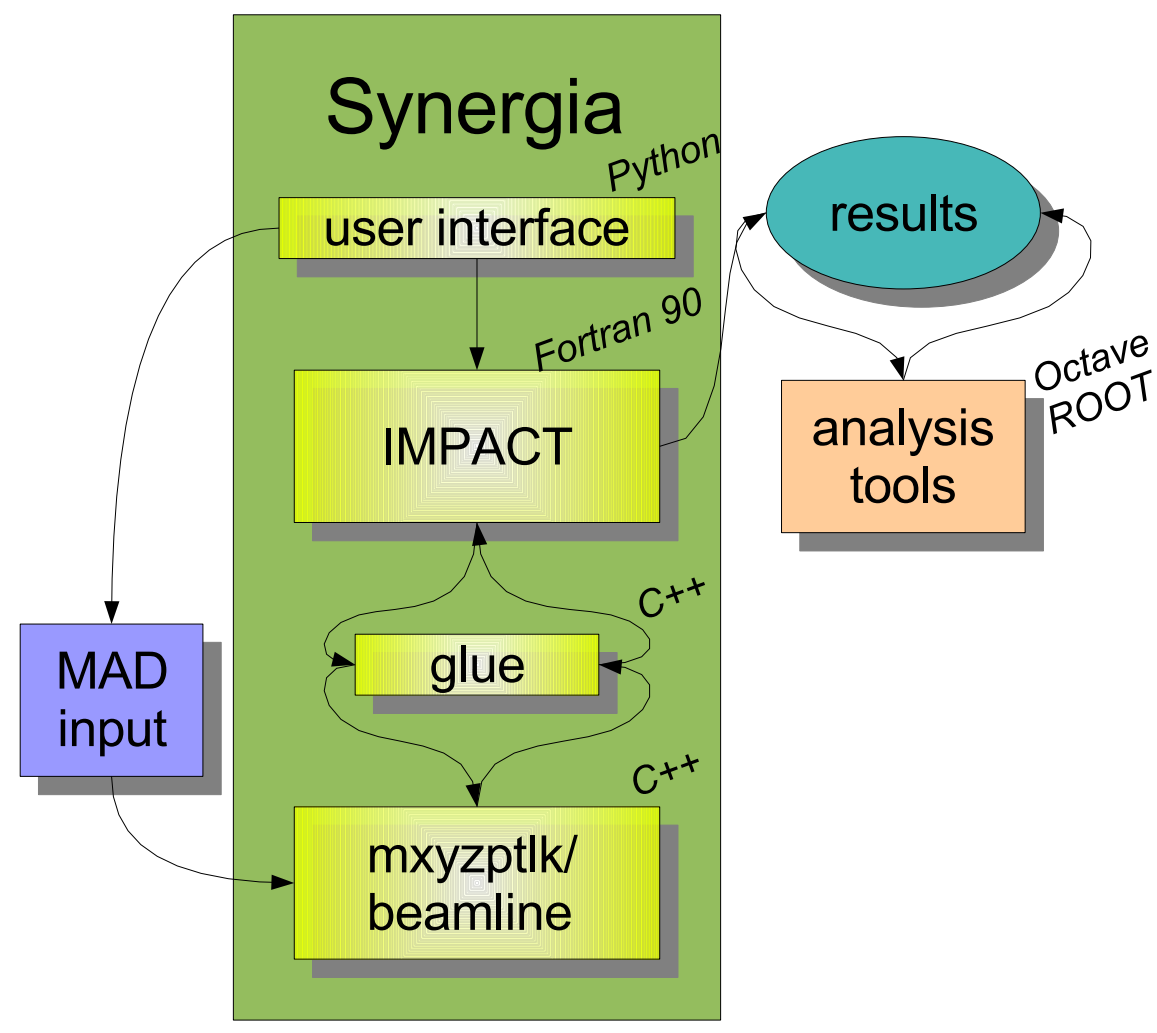

FIG. 1: Synergia components and their relation to outside inputs.

A desirable feature of the mxyzptlk/beamline package for our purposes is the ability to read accelerator descriptions in the MAD8 language [9]. Synergia passes a MAD8 file and lattice name to beamline which returns transfer maps for an arbitrary number of lattice slices. The MAD8 parser in beamline is limited to processing accelerator lattice descriptions since the Synergia interface is much more flexible than the MAD8 command language. In a generic Synergia run lattice elements from MAD8 files can be combined in arbitrary ways and even mixed with native IMPACT/Synergia elements. Synergia also takes advantage of mxyzptlk/beamline's arbitrary-order transfer maps. The current implementation utilizes first- and second-order maps, but generalization to arbitrary orders is planned for the near future.

\section{SYNERGIA}

Synergia is the combination of IMPACT, the mxyzptlk/beamline libraries, glue code to get the two packages talking to each other, and a user interface wrapper providing a straightforward, yet powerful, human interface. Figure 1 shows the relationship between Synergia components, MAD8 files, and analysis tools.

\section{A. Build System}

Portability has been a major design concern in creating Synergia. We rely on multiple components written in multiple languages. While using multiple components allows us to 
quickly put together a powerful package, it also creates a configuration management problem. Multiple-language issues are particularly problematic because calling conventions vary from platform to platform. We solve the multiple language part of the problem by writing all of the inter-language wrapper code in terms of macros that can be redefined for various platforms. We solve configuration management problem by incorporating a modern build system based on the GNU Autotools to provide consistent builds on all platforms.

In principle, building Synergia is as simple as executing "./configure \&\& make \&\& make install" in the mxyzptlk directory followed by "./configure \&\& make" in the Synergia directory. In practice, many options to configure are available. The two principles we have followed in constructing the build system are (1) modifying the source (including Makefiles) should never be necessary, and (2) all options should come with reasonable defaults. To date, Synergia builds without modifications on Linux systems using either the Portland Group F90 compiler or the Intel F90 compiler, g++ or Intel CC, and either the MPICH or LAM implementations of the Message Passing Interface (MPI). Synergia also builds without modifications on AIX, using XL Fortran, Visual Age C++ and POE.

\section{B. Human Interface}

The user-level interface to Synergia consists of a set of Python classes that wrap the low-level interfaces to the code. To run Synergia, the user writes a short Python script utilizing these classes. Example script excerpts are shown in Figures 2 and 3. The use of Python has several advantages: There is no specialized syntax to learn. A user familiar with Python will be able to understand the entire interface easily. A user unfamiliar with Python will be able to copy an example script and modify it with little difficulty. Although most examples will only use Python trivially, the full power of the language is available should it be needed. Last, but not least, the use of an existing scripting language greatly simplifies the implementation, minimizing both the development time and the probability for introducing bugs.

\section{Job Description}

Every Synergia job is a simple Python script. Synergia provides the class Impact_parameters as an interface to the internal parameters of IMPACT, including input beam, energy, space-charge parameters, etc. The accelerator lattice can be defined using elements from an external MAD8 file.

Synergia provides a simple matching module to generate matched beams, utilizing linear optics calculations from mxyzptlk/beamline for lattice function determination. We also provide an interface to our Octave utilities package that generates a matched beam in the presence of space charge by solving the r.m.s. envelope equations.

\section{Job Creation and Submission}

Synergia jobs can be arbitrarily complex. Typically, the user will want to run several different jobs varying only a few of the many input parameters. Synergia provides several facilities to assist the user in creating, submitting and managing simulation runs. 


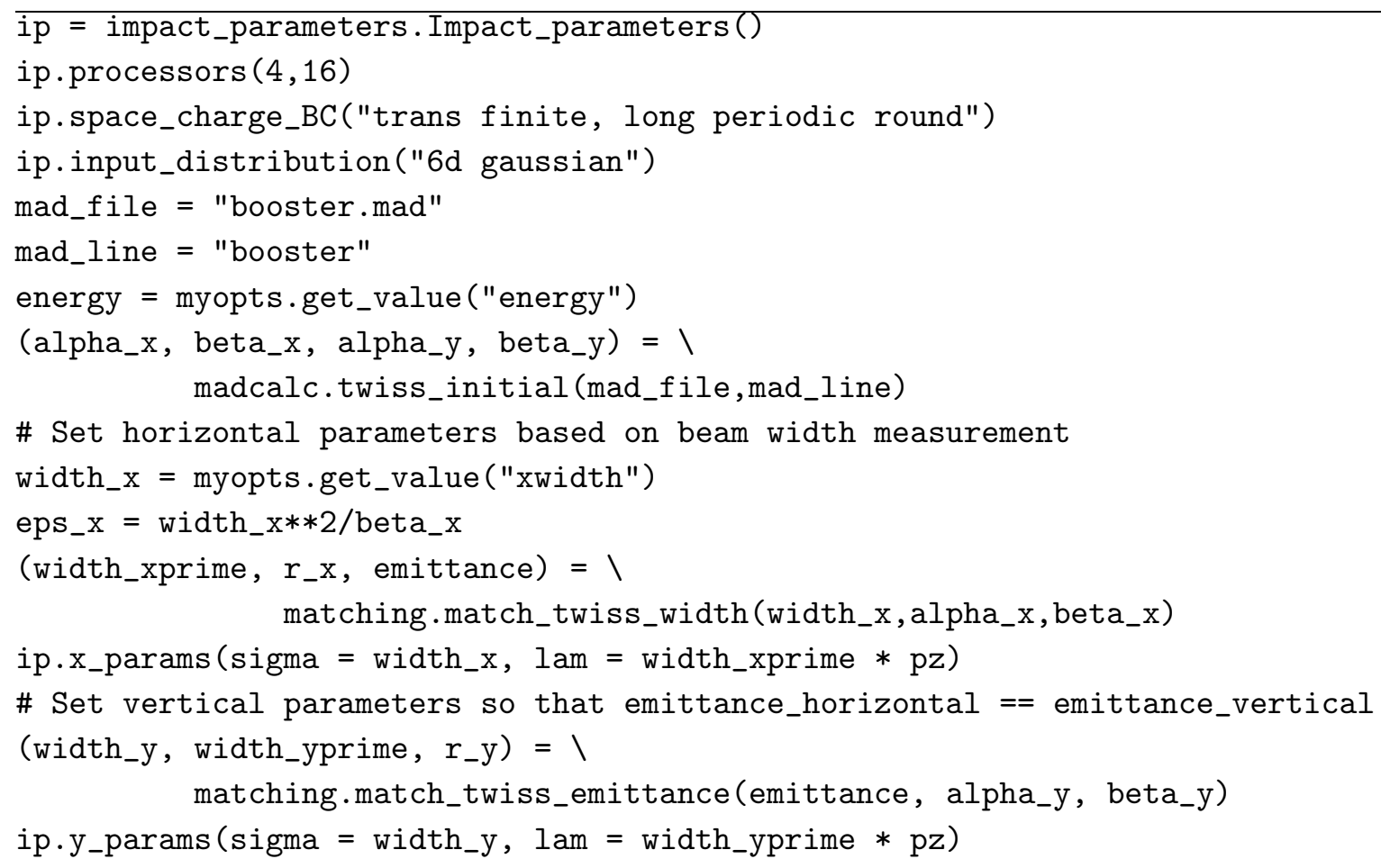

FIG. 2: Example excerpt of a Synergia Python script showing lattice description from MAD8 file and beam matching utilizing Synergia's matching module.

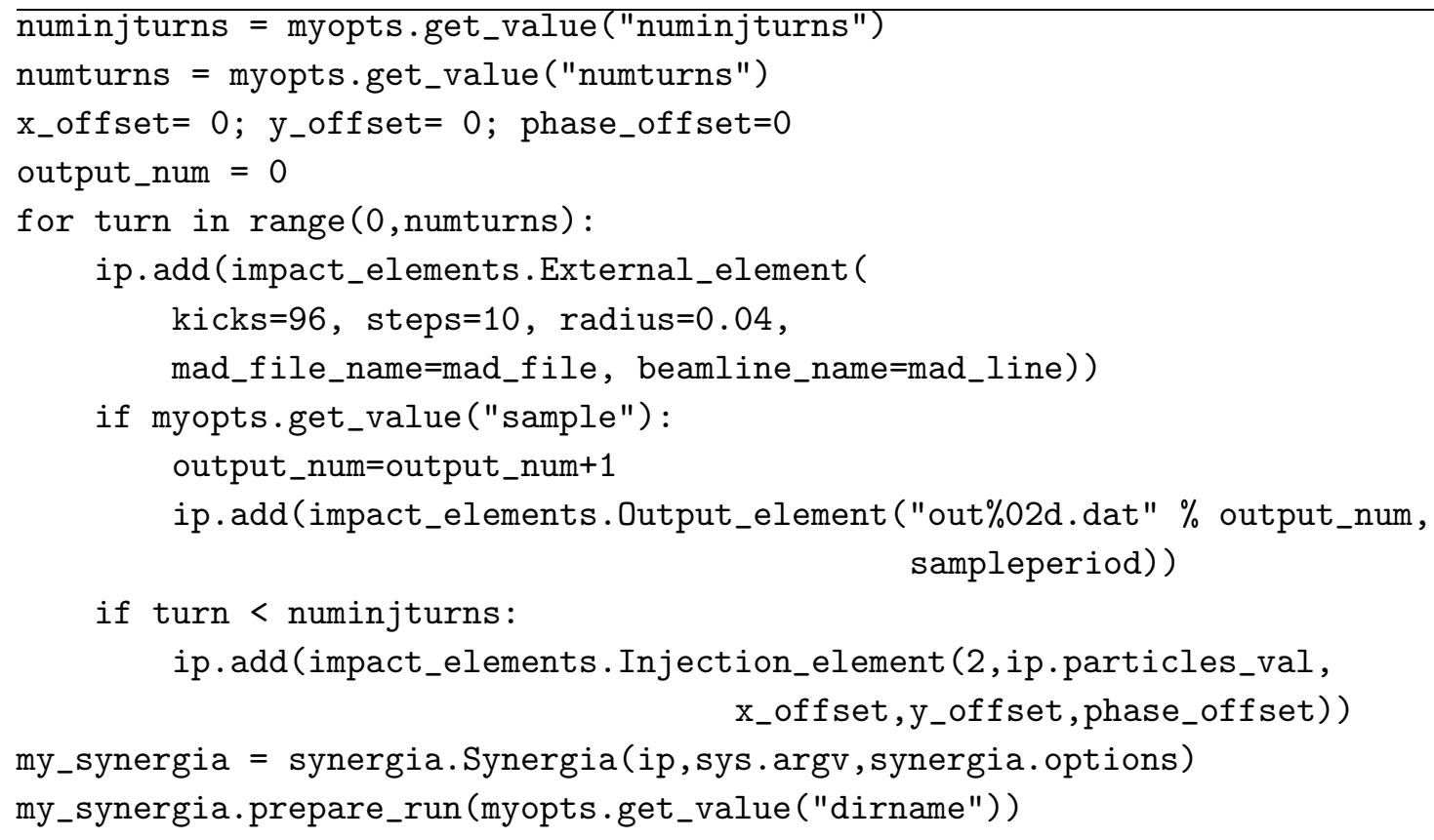

FIG. 3: Example excerpt of a Synergia Python script specifying a simulation with multiple injection turns utilizing basic Python. 
A Python module options provides a simple method to write scripts accepting commandline arguments for Synergia and user-defined parameters. When a Synergia job script is run, the command-line options for that job are automatically recorded in a manner so that they can be edited and/or reinvoked. Synergia also records all job parameters in a humanreadable description file.

Synergia automatically generates batch system submission scripts based on a usersupplied template. Several example templates are provided, including templates for singleprocessor machines, multi-processor machines, the PBS batch system and more. Optionally, jobs can be defined to run on remote machines. Synergia generates scripts to export the input files to the remote machine, submit the job, and retrieve the files from the remote machine once the job is finished.

\section{Diagnostics}

A number of diagnostics are provided by default during the simulation run. In addition, we provide tools to allow users to analyze simulated data after a simulation has completed. The standard diagnostic utilities are evaluated at each split-operator step and include calculations of the second, third and fourth moments of all six degrees of freedom, two-, four-, and six-dimensional emittances, and all pairwise correlations for beam components.

For post-processing, we provide the ability to dump the entire beam, or a sampled subset of the beam, at any simulation step. Files can be dumped in plain text or HDF5 format [10]. Each particle is saved along with a unique tag so that individual particles can be tracked throughout the simulation. We provide tools for rearranging a series of particle dumps into individual tracks, both for diagnostic purposes and calculating particle tunes. The output format of the particle information dumps can be easily interfaced to visualization packages such as OpenDX [11]. An example of such visualization of a FNAL Booster simulation is shown in Figure 4.

\section{Distributions with general correlations}

Synergia can generate Gaussian beams with arbitrary two-component correlations. Generation of random distributions with finite statistics leads to statistical errors in the moments of the generated distributions. We use, typically, $\mathcal{O}\left(10^{6}\right)$, macroparticles to simulate $\mathcal{O}\left(10^{12}\right)$ real particles. The statistical errors in the simulation are therefore an artifact of the simulation only. We correct for these errors at the level of two-component correlations in the following procedure.

We want to generate a set of random vectors $\{r\}$ such that

$$
\left\langle r_{j}\right\rangle=\bar{r}_{j}
$$

and

$$
\left\langle r_{j} r_{k}\right\rangle=C_{j k},
$$

where $\bar{r}_{j}$ and $C_{j k}$ are given. A typical application would be a beam with no offset $\left(\bar{r}_{j}=0\right)$ and $C_{j k}$ chosen to create a matched beam. We start by generating a finite set of (nominally) uncorrelated random vectors $\{\rho\}$. These vectors will have first and second moments

$$
\bar{\rho}_{j} \equiv\left\langle\rho_{j}\right\rangle
$$




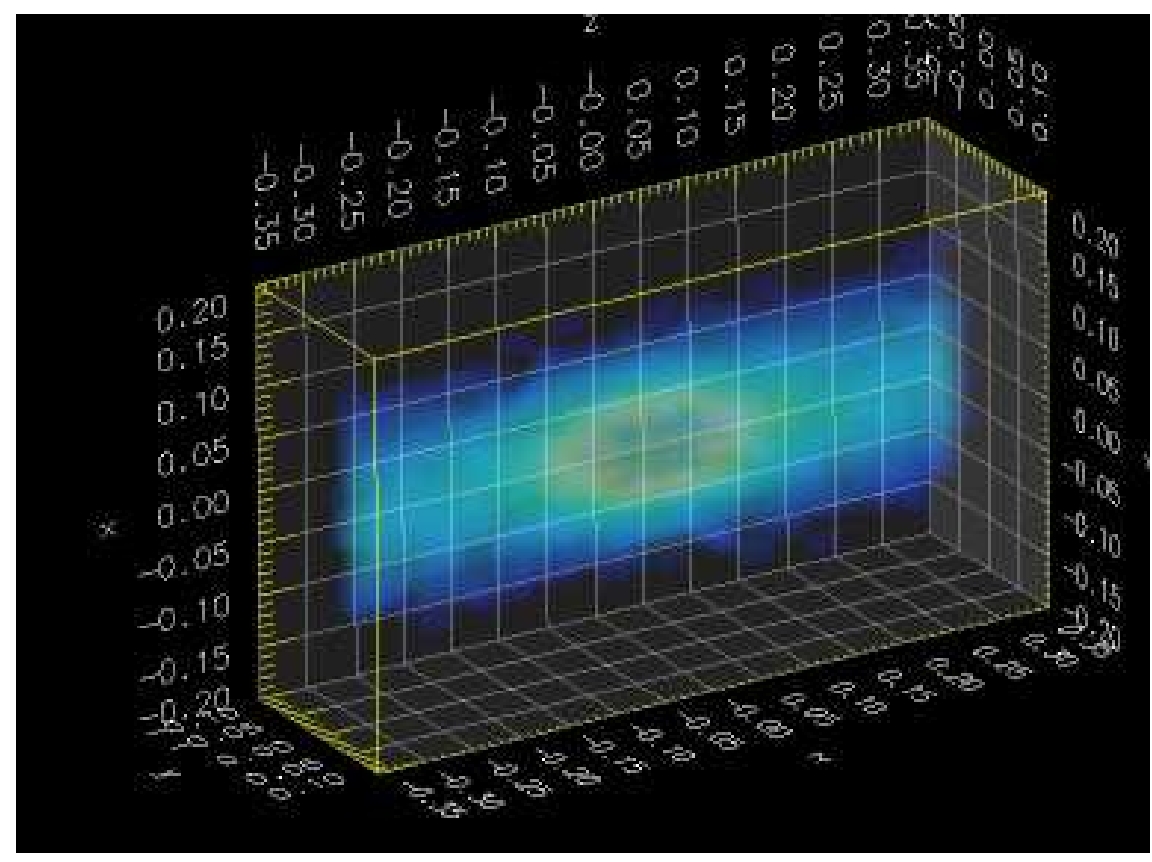

FIG. 4: OpenDX visualization of a three dimensional histogram of particle density of a FNAL Booster simulation.

and

$$
X_{j k} \equiv\left\langle\rho_{j} \rho_{k}\right\rangle .
$$

In the limit of infinite statistics, $X_{j k} \rightarrow \delta_{j k}$. Finite effects cause deviations from this limit, which will introduce small, but unphysical, deviations from the desired distribution. In order to correct for these effects, we use the transformation

$$
r_{j}=A_{j k}\left(\rho_{k}-\bar{\rho}_{k}\right)+\bar{r}_{j}
$$

Where

$$
\begin{aligned}
& C=G G^{T}, \\
& X=H H^{T},
\end{aligned}
$$

and

$$
A=G H^{-1} \text {. }
$$

The resulting set of vectors $\{r\}$ will have the correct first and second moments, with no error contribution due to finite statistics.

\section{Multi-turn injection}

Synergia provides an injection module to allow modeling of multi-turn injection in a completely transparent manner. The injection module generates additional macro-particles according to a given distribution. The total beam current represented by these macroparticles is a parameter. An injection "element" can be placed anywhere between beamline elements in a given lattice, thus multi-turn injection can be simulated by simply including such an element in a loop. (See Figure 3). In our implementation, both the number of 
macro-particles and the beam current increase with any subsequent use of the injection module. To allow for injection painting, the injection module includes in its argument list vertical, horizontal, and longitudinal phase offsets for the center of the beam distribution.

\section{PARALLEL PERFORMANCE}

We have run benchmarks of our code on four different clusters under a variety of configurations. Our benchmark is a simulation of a single revolution of the FNAL Booster (see Section V.) The simulation included 2.7 million particles undergoing 100 space-charge kicks on a $65 \times 65 \times 65$ grid.

Three of the clusters are Linux clusters: lqcd [17], heimdall [18] and Alvarez [19]. Our benchmarks include a sampling of the range of currently-available networking options for Linux: 100 Mbit Ethernet, Gigabit Ethernet and Myrinet 2000. We also compared the performance of the Intel fortran compiler (ifc) with the Portland Group fortran compiler (pgf90). For the former, the code was compiled with the optimization setting "-02". For the latter the code was compiled with the setting "-fast". The fourth cluster we used for benchmarking was Seaborg [20], the 6,080-processor IBM SP at NERSC.

The results of our benchmarks are displayed in Figure 5. Overall, we find that Synergia scales very well up to a certain scale set by the networking used in each case. The clear winner in scaling is the specialized configuration found in Seaborg. The fastest Linux clusters, however, showed overall superior performance. We can also see that Gigabit or Myrinet is necessary for a Linux cluster to effectively take advantage of more than a few processors. These tests were insufficient to distinguish between Gigabit and Myrinet. Somewhat surprisingly, we also see that the Intel compiler produced significantly better performance than the Portland compiler for our application.

Since for most medium-size applications we use the qcd80 cluster [21], we have optimized the performance of our simulations for this machine. Figure 6 shows the scaling performance we obtained on qcd80, for a FNAL Booster simulation on a $33 \times 33 \times 257$ grid. We obtain better scaling behavior in this case because we have optimized for the particular cluster and simulation.

\section{SYNERGIA TESTS AND APPLICATIONS}

In order to verify the accuracy of our simulation we model several cases simple enough to perform comparisons with semi-analytic calculations. We start with the case of a K$\mathrm{V}$ beam distribution in an idealized FODO channel[23]. Then, we compare the Synergia prediction for the evolution of the second moments of a Gaussian beam distribution in a FODO channel to the solution of the envelope equation. We also compare FODO channel results from Synergia with another space-charge code. Finally, we compare the tune shifts predicted by Synergia to that of the Laslett tune shift formula [14].

The first realistic application of Synergia has been to model the FNAL Booster [5] during the first few hundred turns after injection. First, we study the incoherent tune shifts for different beam currents. Then, we study patterns of halo formation quantitatively, as well as qualitatively. Finally we examine emittance growth in various beam configurations. 


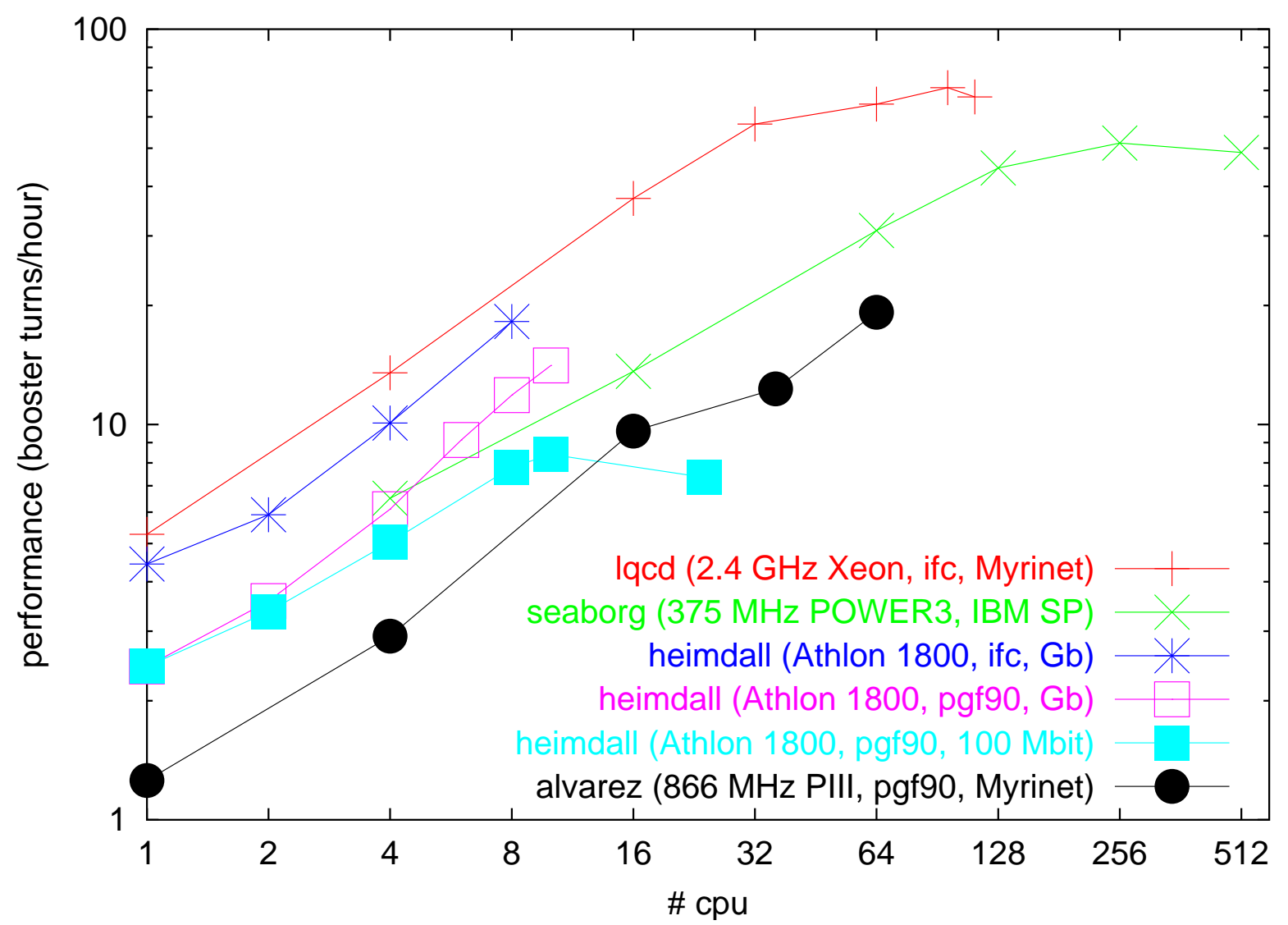

FIG. 5: Performance, defined as simulated Booster turns per hour, versus the number of processors used, on various parallel machines.

\section{A. Synergia Benchmarking}

For a $\mathrm{K}-\mathrm{V}$ distribution the charge density across the beam is constant and the forces associated with space charge vary linearly with the coordinates $x$ and $y$. The evolution of the beam envelope can be calculated exactly by integrating the envelope equations [22]. As a first check, we compare the evolution of a K-V beam as predicted by Synergia to the solution of the envelope equations:

$$
\sigma_{x}^{\prime \prime}+K_{x} \sigma_{x}-\frac{\epsilon_{\mathrm{rms}}^{2}}{\sigma_{x}^{2}}=\frac{\xi}{4\left(\sigma_{x}+\sigma_{y}\right)}
$$

and

$$
\sigma_{y}^{\prime \prime}+K_{y} \sigma_{y}-\frac{\epsilon_{\mathrm{rms}}^{2}}{\sigma_{y}^{2}}=\frac{\xi}{4\left(\sigma_{x}+\sigma_{y}\right)},
$$

where $\xi=4 Q^{2} r_{0} \lambda /\left(A \beta^{2} \gamma^{3}\right)$, with $Q$ the charge of a beam particle in units of $e, r_{0}$ is the classical proton radius, $\lambda$ is the line charge density, $A$ is the atomic number, $K_{x / y}$ are the focusing strengths, $\sigma_{x}=\left\langle x^{2}\right\rangle^{1 / 2}, \sigma_{y}=\left\langle y^{2}\right\rangle^{1 / 2}$, and $\epsilon_{\mathrm{rms}}$ is the unnormalized r.m.s. emittance:

$$
\epsilon_{\mathrm{rms}}=\left\langle x^{2}\right\rangle\left\langle p^{2}\right\rangle-\langle x p\rangle^{2}=\frac{\left\langle x^{2}\right\rangle}{\beta_{\text {Twiss }}} .
$$




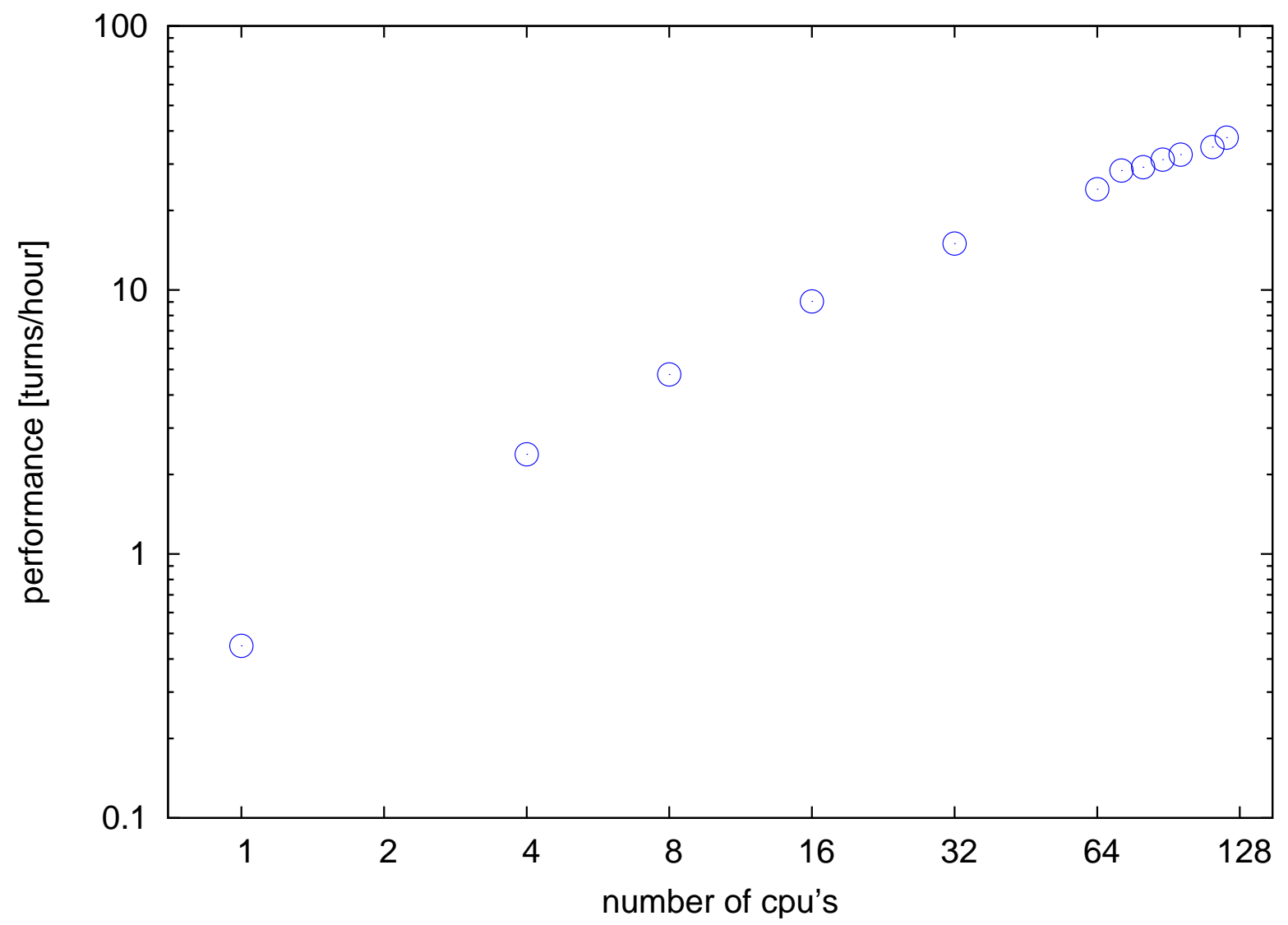

FIG. 6: Performance, defined as simulated Booster turns per hour, versus the number of processors used, on qcd80.

Note that the r.m.s. value of $x$ in a K-V beam of radius $a$ is given by $\left\langle x^{2}\right\rangle=a^{2} / 4$.

In Figure 7 we compare the numerical solution of Equations 11 and 12 to the Synergia result for the FODO channel defined by the following MAD8 file:

scale $=0.374749636$

drs: drift, $1=7.44 \mathrm{~d}-2$

drl: drift, $l=14.88 \mathrm{~d}-2$

qd7: quadrupole, $1=6.10 \mathrm{~d}-2, \mathrm{k} 1=-38.64 \mathrm{~d} 0 / \mathrm{scale}$

qf7: quadrupole, $1=6.10 \mathrm{~d}-2, \mathrm{k} 1=38.64 \mathrm{~d} 0 / \mathrm{scale}$

channel: line $=(\mathrm{drs}, \mathrm{qd} 7, \mathrm{drl}, \mathrm{qf} 7, \mathrm{drs})$

For this comparison we used a $\mathrm{K}-\mathrm{V}$ beam with a kinetic energy $0.0067 \mathrm{GeV}$ and two dimensional transverse emittance $3.1 \times 10^{-6} \mathrm{~m}$ rad in both the horizontal and vertical planes. Figure 7.a shows the comparison of the calculated horizontal beam width for a matched beam of 0.5 Amps. Figure 7.b shows the effects of taking into account space charge in the matching procedure in the evolution of the horizontal beam width. The Synergia prediction is consistent with the numerical solution of the envelope equations. The differences between the curves in Figure 7.b are a measure of the magnitude of the space-charge effect.

In the case of a more realistic beam distribution, such as a Gaussian distribution, the envelope equations can model the evolution of the second moments of the beam distribution under the assumption that the emittance evolution is known [22]. In the cases presented here 


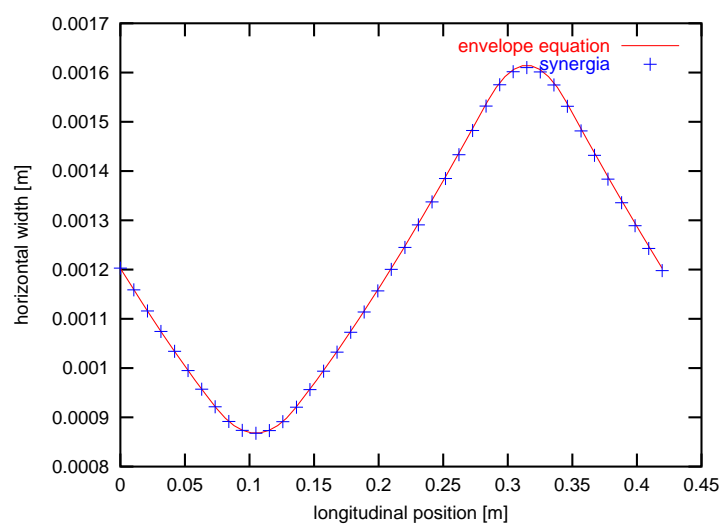

(a)

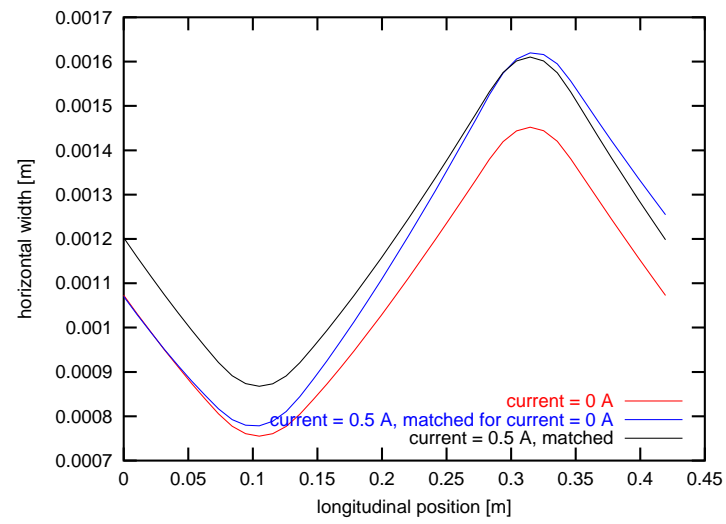

(b)

FIG. 7: (a) Comparison of the Synergia prediction for the horizontal beam evolution in the FODO lattice described in the text to the solution of the envelope equations. (b) Effect of including space charge in the matching condition, as predicted by Synergia.

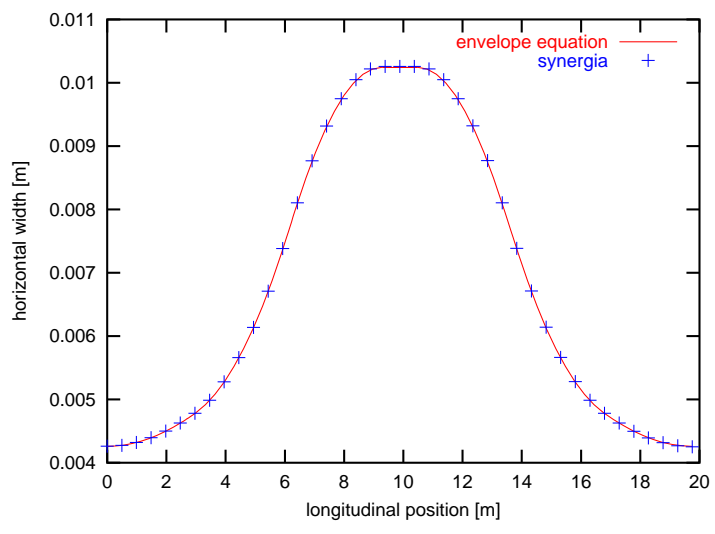

(a)

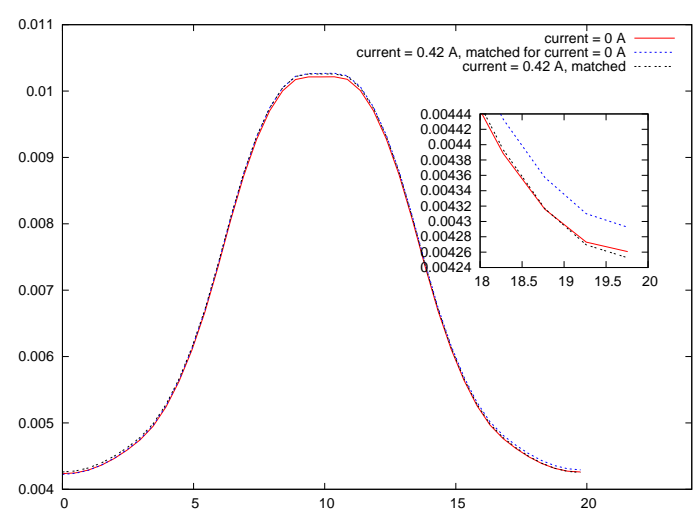

(b)

FIG. 8: (a) Comparison of the beam width evolution in a FNAL Booster cell as predicted by Synergia and the solution of the envelope equations. (b) Effects of space charge on matching, as predicted by Synergia. The insert shows the detail of these effects at the end of the cell.

we assume that the emittance remains constant. We compare the prediction of Synergia with the prediction of the envelope equations for the evolution of the width of a Gaussian beam in a lattice cell of the FNAL Booster [5]. Here we use a beam that is Gaussian in the transverse coordinates and uniform in the longitudinal coordinate. The results are shown in Figure 8.a. In Figure 8.b we show the effects of including space charge in the matching condition, as predicted by Synergia. The current used in this simulation is a typical operating current for the machine. In this case the space-charge effect is small for the r.m.s. width change in one Booster cell. Traversing a single cell is a tiny fraction of the entire cycle in which the beam passes through 480,000 cells.

As our final exercise in modeling beam envelopes, we compare Synergia with the MaryLie/IMPACT (ML/I) code [6]. The comparison is done for the FODO channel de- 


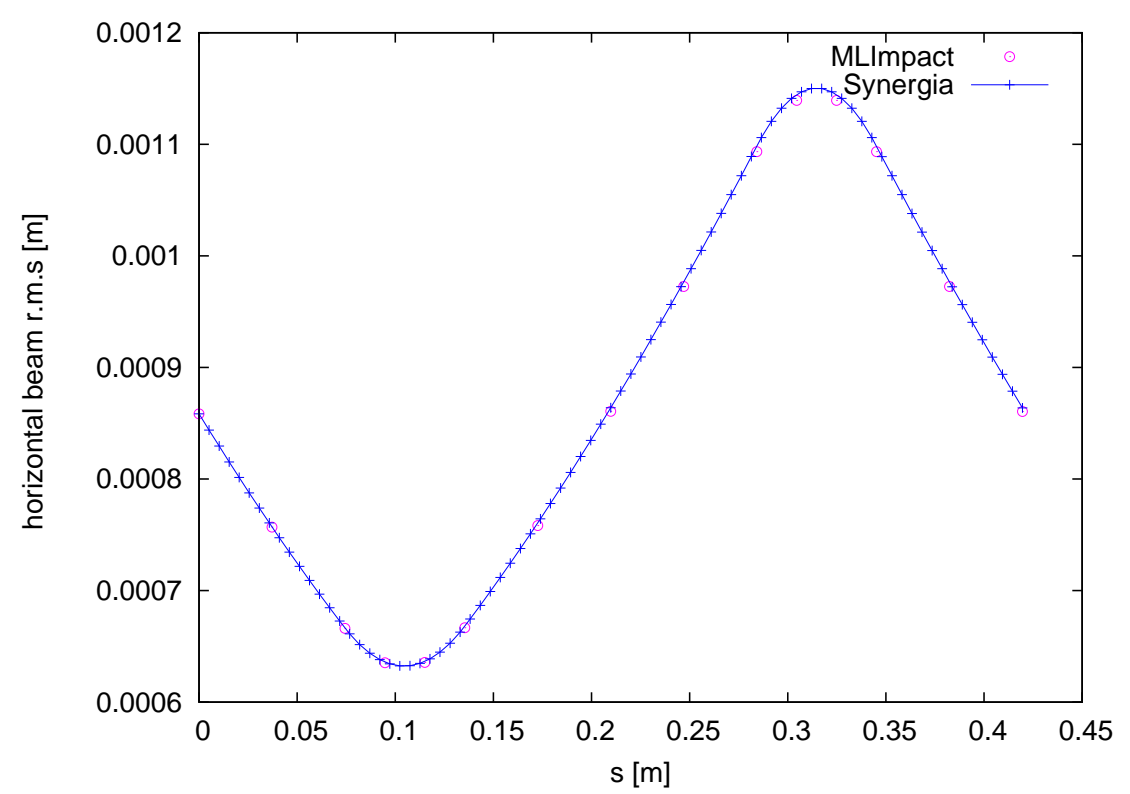

FIG. 9: Comparison of the Synergia and MaryLie/IMPACT predictions for the horizontal r.m.s. beam size of a $\mathrm{K}-\mathrm{V}$ beam propagating in the FODO channel described in the text as a function of $s$.

scribed above, using a 0.5 Amps matched $\mathrm{K}-\mathrm{V}$ beam with two-dimensional transverse emittance of $1.0 \times 10^{-6} \mathrm{~m}$ rad in both planes. For this comparison we used a common input file of beam particles for both the Synergia and ML/I simulations. In Figure 9 we show the comparison of the horizontal r.m.s. beam size predictions from the two codes. The agreement is very good. The difference between the prediction of the two simulations for the r.m.s. width of the beam at the end of the channel is $0.27 \%$. This slight variation in the final answer is due to minor differences in the implementation of the Poisson solver and differences in the problem description in the simulation, such as the number of slices used in the split-operator particle advance algorithm.

Another simple comparison we can make with analytic calculations is to compare the Laslett tune shift for a $\mathrm{K}-\mathrm{V}$ beam with results from a Synergia simulation. We use the formula

$$
\Delta \nu=\frac{-N r_{0}}{8 \pi \beta^{2} \gamma^{3} \epsilon_{\mathrm{rms}}},
$$

where $N$ is the number of particles in the beam, $r_{0}$ is the classical proton radius and $\epsilon_{\mathrm{rms}}$ is the unnormalized r.m.s. emittance as defined in Equation 13. The Synergia prediction for the tune shift is obtained by taking the peak of the Fourier transform of the horizontal and vertical position of individual particles, as a function of $s$, sampled each cell (24 times per turn) for 100 turns. Here $s$ is the coordinate along the path of the reference trajectory. By sampling each cell, we are able to extract the integer portion of the tune. Sampling once per turn is sufficient to extract the fractional tune. In Figure 10 we show the comparison between the results from Equation 14 and Synergia for the FNAL Booster ("bare" lattice, as described above) and for different beam currents. The agreement is very good; we thus conclude that Synergia can reliably reproduce analytical calculations of space-charge effects. 


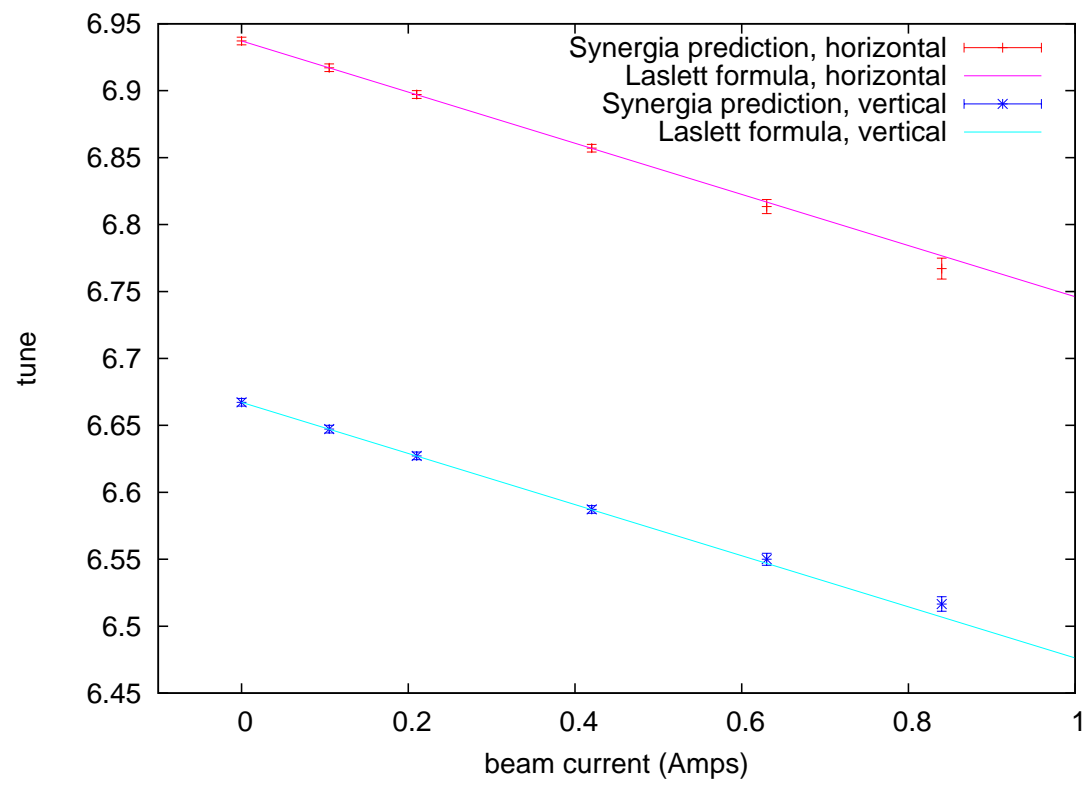

FIG. 10: Comparison of the horizontal and vertical tune shifts calculated using Synergia and using equation 14.

\section{B. Application to the FNAL Booster}

In this section we present initial results from Synergia studies of beam behavior in the FNAL Booster during the first few hundred turns after injection. A more detailed study of the interplay between single particle optics effects and space-charge effects will follow in a separate publication.

The Booster is a rapid-cycling, $15 \mathrm{~Hz}$, alternating gradient synchrotron with a radius of 75.47 meters. The lattice consists of 96 combined function magnets in 24 periods, with nominal horizontal and vertical tunes of 6.9 and 6.7 respectively. The Booster accelerates protons from a kinetic energy of $400 \mathrm{MeV}$ to $8 \mathrm{GeV}$, at harmonic number $h=84$, using 17 RF cavities with frequency that slews from $37.7 \mathrm{MHz}$ at injection to $52.8 \mathrm{MHz}$ at extraction. The nominal average current at injection is $\sim 42 \mathrm{~mA}$. Typically, the injection process lasts for ten Booster turns. The injected beam is a stream of bunches equally spaced at the linac [12] RF frequency of 201.2 MHz.

There are many factors affecting the behavior of the Booster beam, including the energy spread and emittance of the injected beam, nonlinear field errors and space-charge effects. The space-charge effects have long been believed to be responsible for a significant fraction of the observed losses in the Booster [13] during the first $2 \mathrm{~ms}$ of the cycle (the injection, capture, and bunching phases). In this section we present a rudimentary study of the these effects in an idealized Booster; we will examine these effects in greater detail in a subsequent paper. For all of the calculations in this paper we have used an idealized "bare" Booster lattice without any non-linear elements. We defer the inclusion of effects such as magnet offsets, correctors, etc., to a future study.

As our first example, we consider the transverse tune spread due to space charge, using a Booster lattice without nonlinear beamline elements and without the complications of multi-turn injection, but with realistic input beam parameters. The initial beam used in 
the simulation is a 6-dimensional Gaussian distribution, with the appropriate correlations to match it to the Booster lattice, accounting for space-charge effects. The horizontal and vertical r.m.s. emittance was $3.05 \times 10^{-6} \mathrm{~m}$ rad. The full current was injected in a single turn and second order maps were used for the single particle optics. The momentum spread, $\Delta p / p$, was 0.0003 . We used 96 space-charge kicks per turn, calculated on a $33 \times 33 \times 257$ computational grid with an average of four particles per grid cell. We followed the beam for 100 turns after injection, recording particle information once per space-charge kick. In this comparison, we used currents of $0,0.105,0.210,0.420,0.630$ and 0.840 Amps. As stated previously, the nominal Booster current is 0.420 Amps.

We compare the Synergia results with the results from the Laslett tune shift formula [14] for a Gaussian beam,

$$
\Delta \nu=\frac{-N r_{0}}{4 \pi \beta^{2} \gamma^{3} \epsilon_{\mathrm{rms}}} .
$$

Note that the above tune shift for a Gaussian beam is a factor of two larger than the tune shift for a K-V beam in Equation 14.

In order to quantitatively study the spread of tune shifts in the two-dimensional transverse tune space, we introduce the concept of generalized two-dimensional r.m.s. ellipse. The ellipse is given by taking the covariance matrix

$$
C=\left(\begin{array}{cc}
\left\langle x^{2}\right\rangle-\langle x\rangle^{2} & \langle x y\rangle-\langle x\rangle\langle y\rangle \\
\langle x y\rangle-\langle x\rangle\langle y\rangle & \left\langle y^{2}\right\rangle-\langle y\rangle^{2}
\end{array}\right),
$$

decomposing into

$$
C=R R^{T}
$$

where $\mathrm{R}$ is lower diagonal. The ellipse is then parameterized by

$$
\left(\begin{array}{l}
x \\
y
\end{array}\right)=R\left(\begin{array}{c}
\sin \theta \\
\cos \theta
\end{array}\right)+\left(\begin{array}{c}
\langle x\rangle \\
\langle y\rangle
\end{array}\right) .
$$

For the case of tune spreads, we take $x$ to be the horizontal tune and $y$ to be the vertical tune. The resulting r.m.s ellipse is a model-independent, statistically robust representation of the spread of the majority of the particle tunes.

The results for the different beam currents are summarized in Figure 11, which shows the transverse tune spread together with the corresponding generalized two-dimensional r.m.s. ellipses of the horizontal and vertical tunes. The tune spread is obtained by taking the peak of the Fourier transform of the horizontal and vertical position of individual particles, as a function of $s$, sampled 24 times per turn for 100 turns. Note that the tune footprint plot shows the extent of the distribution but not the density of the points, which is better represented by the two-dimensional r.m.s. ellipses. The filled squares in the figure correspond to the prediction from the Laslett formula for the corresponding beam current ("nominal" refers to the tune prediction for the zero current case).

The generalized two-dimensional r.m.s. ellipse

Since the Laslett formula predicts tunes shifts for particles at the core of a stationary beam its prediction is an upper limit of what we observe in the self-consistent particle simulation. Particles away from the center of the distribution experience smaller space-charge forces.

As a second example, we study the formation of halo in the case of mismatched beam. In Figure 12 we plot the kurtosis[24]

$$
k \equiv \frac{\left\langle(x-\langle x\rangle)^{4}\right\rangle}{\left\langle(x-\langle x\rangle)^{2}\right\rangle^{2}}-3
$$




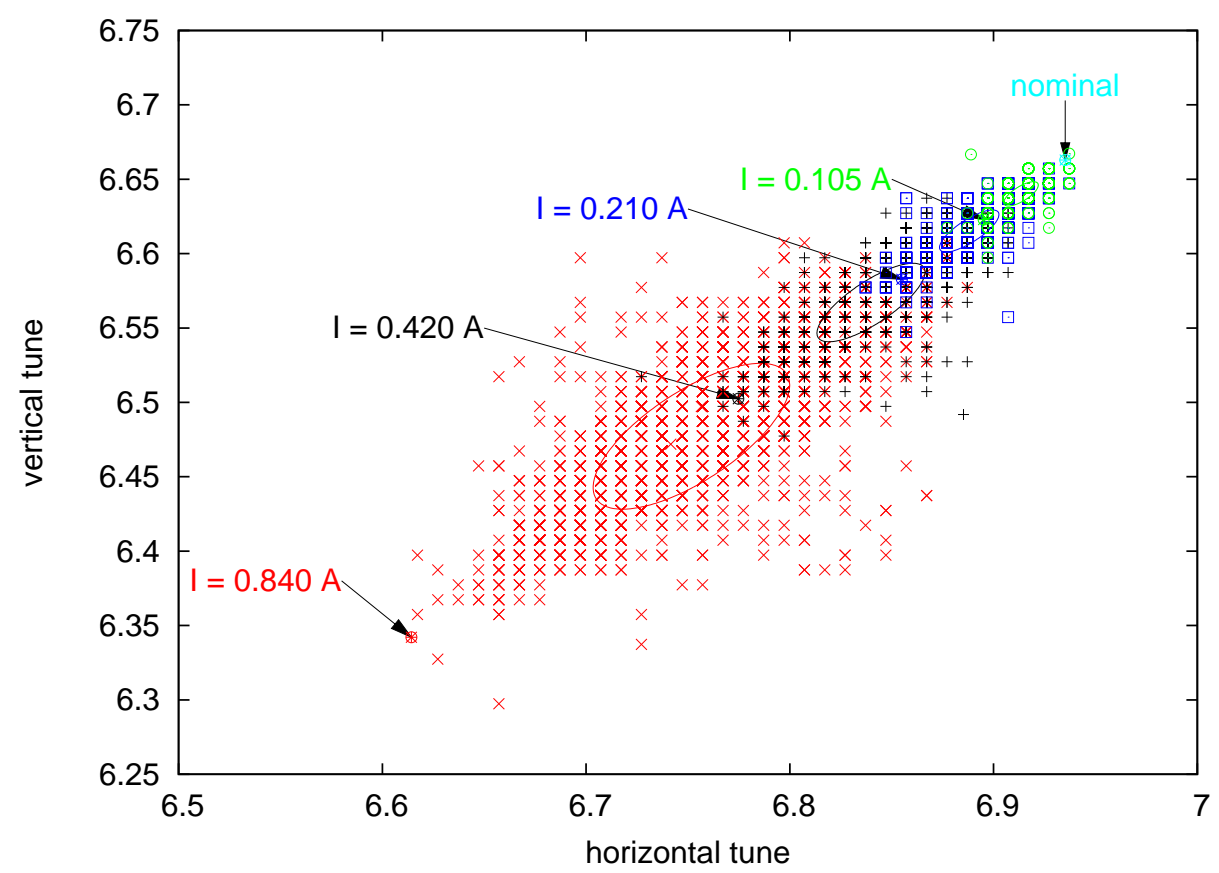

FIG. 11: Transverse tune spread calculated by Synergia, compared to the Laslett tune shift formula. The green circles correspond to a current of 0.105 Amps, the blue square symbols to 0.210 Amps, the black stars to 0.420 Amps and the red x's to 0.840 Amps. The definition of the ellipses is given in the text. The arrows point to the tune predicted by the Laslett formula for each current.

of the beam distribution in each transverse plane as a function of $s$. The beam parameters are as described above, with a beam current of 0.420 Amps. We ran four different cases, varying the initial beam conditions: matched beam with and without momentum spread for single-turn injection, and $20 \%$ mismatched beam with momentum spread $(\Delta p / p=0.0003)$ for single- and multi-turn injection. For these simulations the lattice does not include any non-linear elements, but since we use second order transfer maps we expect chromatic effects to contribute to halo creation for non-zero momentum spread. In the cases with mismatch, the beam has been mismatched in both planes by stretching the width by a factor of 1.2 and adjusting the conjugate momentum distribution to maintain the original emittance.

We observe that in the matched beam cases, the kurtosis is close to zero and that non-zero momentum spread has a small effect in the horizontal and no effect in the vertical plane. The reason for the small difference in the horizontal is due to our matching procedure: we first match the beam correlations for the presence of dispersion, and then we match for the presence of space charge, neglecting any interaction between the two effects. This results in a small residual mismatch in the horizontal (non-zero dispersion) plane. Note that a Gaussian distribution has $k=0$. A distribution with $k>0$ is known as leptokurtic, while a distribution with $k<0$ is known as platykurtic. In the case of the mismatched beam, the simulation quickly converges to a leptokurtic distribution, an indication of the halo formation. The multi-turn injection case shows a smaller increase in kurtosis than the single-turn case since the space-charge effects turn on gradually, resulting in a painting effect. We note that the authors of Ref. [16] use the same method to identify halo formation, except that they define a new parameter $h$, the "spatial-profile parameter." The spatial-profile parameter is related 


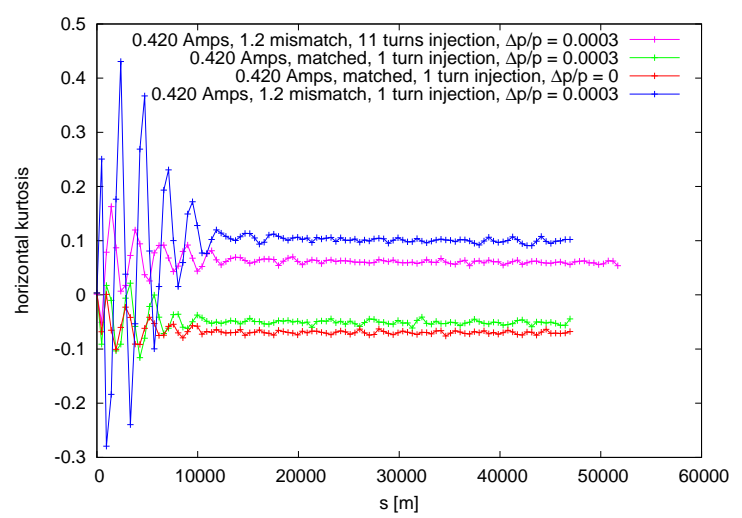

(a)

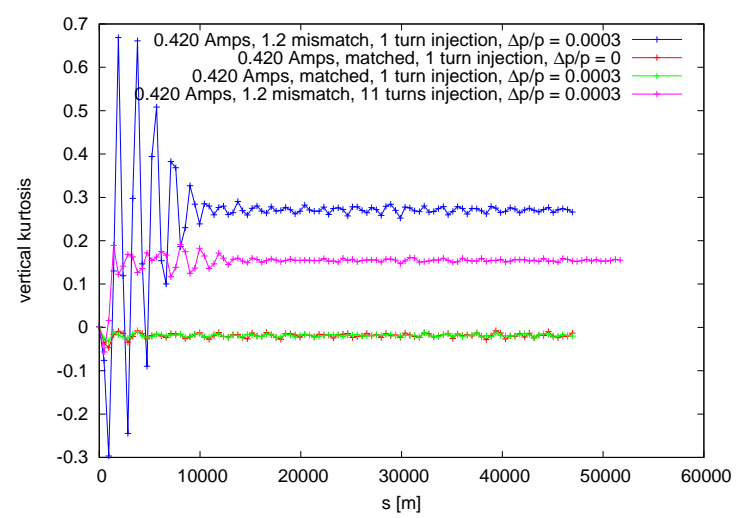

(b)

FIG. 12: Kurtosis in the horizontal (a) and vertical (b) planes, as a function of $s$. The beam current is $0.420 \mathrm{Amps}$, and the beam is matched with $\Delta p / p=0$ (red), matched with $\Delta p / p=0.0003$ (green), and mismatched with a $20 \%$ mismatch in both the horizontal and vertical widths, $\Delta p / p=0.0003$, and for single turn (blue) and 11 turn (purple) injection.

to kurtosis by $h=k+1$.

In order to present a qualitative measure of the amount of halo created in the above simulations, we plot two dimensional phase space projections of the mismatched beam in both the transverse and longitudinal planes. Note that for the transverse phase space plots we use normalized coordinates, where $x$ and $y$ are scaled by $l=c / w$, with $c$ the speed of light and $w$ the angular frequency, and $x^{\prime}$ and $y^{\prime}$ are scaled by $m c$, where $m$ is the proton mass. Figures 13 and 14 show the evolution of the horizontal and vertical phase spaces, while Figure 15 shows the evolution of a slice of the longitudinal phase space. The transverse phase space plots give a qualitative picture of halo formation. The longitudinal phase space plots show the transition from the bunched injected beam through debunching to a DC beam. The simulated injected beam models a realistic Booster beam at injection which is bunched according to the $200 \mathrm{MHz}$ Linac rf. The phase space slice in Figure 15 corresponds to a full period of the $200 \mathrm{MHz}$ rf.

Finally, we investigate how space-charge and chromatic effects affect the emittance of the Booster. In Figure 16 we plot the normalized 4-D transverse emittance[25] for five different initial beam conditions, described in the caption of the figure. As expected, in the cases where the beam was matched there is no emittance growth. That is the case for both zero and non-zero momentum spread, and for space charge. (Our matching procedure takes into account space-charge effects on the second moments of the beam). In the mismatched cases we observe a $12 \%$ increase of the beam emittance during the first 10 to 15 turns after injection. The effect is a combination of chromatic and space-charge effects and it is very similar for both the single- and multi-turn injection cases. The total current is the same, 0.420 Amps, in both cases. 


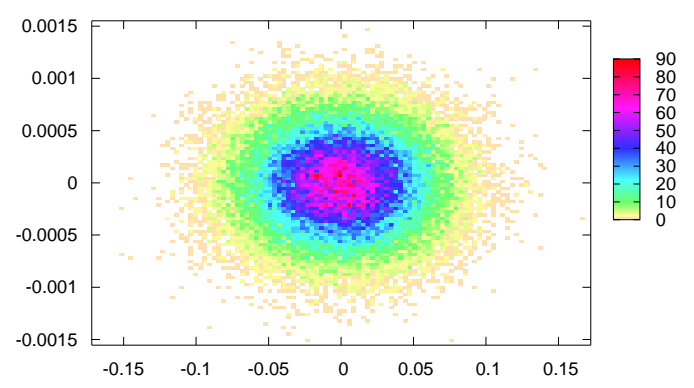

(a)

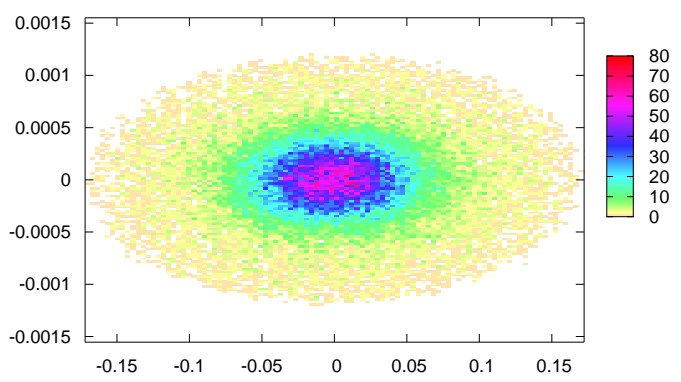

(b)

FIG. 13: Horizontal ( $x$ vs. $x^{\prime}$ ) phase-space plot for the mismatched beam case. (a) Beam in the beginning of the simulation, (b) after 100 turns.

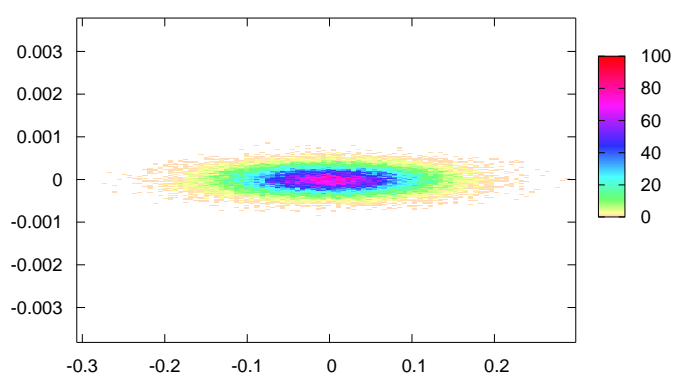

(a)

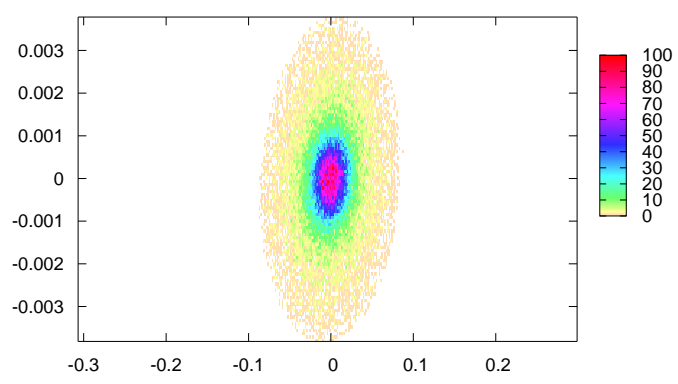

(b)

FIG. 14: Vertical ( $y$ vs. $y^{\prime}$ ) phase-space plot for the mismatched beam case. (a) Beam in the beginning of the simulation, (b) after 100 turns.

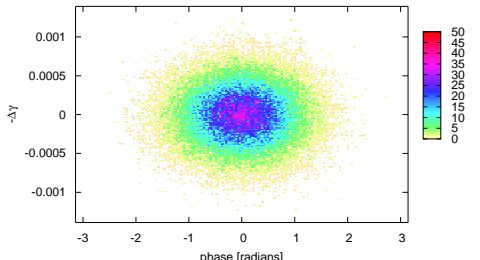

(a)

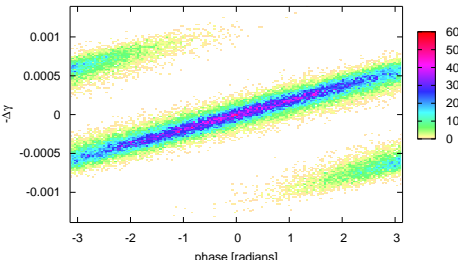

(b)

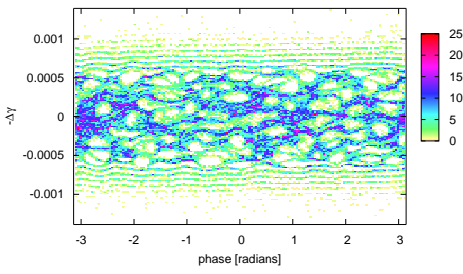

(c)

FIG. 15: Longitudinal (phase vs. $-\Delta \gamma$ ) phase-space plot for the mismatched beam case. (a) Beam in the beginning of the simulation, (b) after 3 turns, and (c) after 100 turns. 


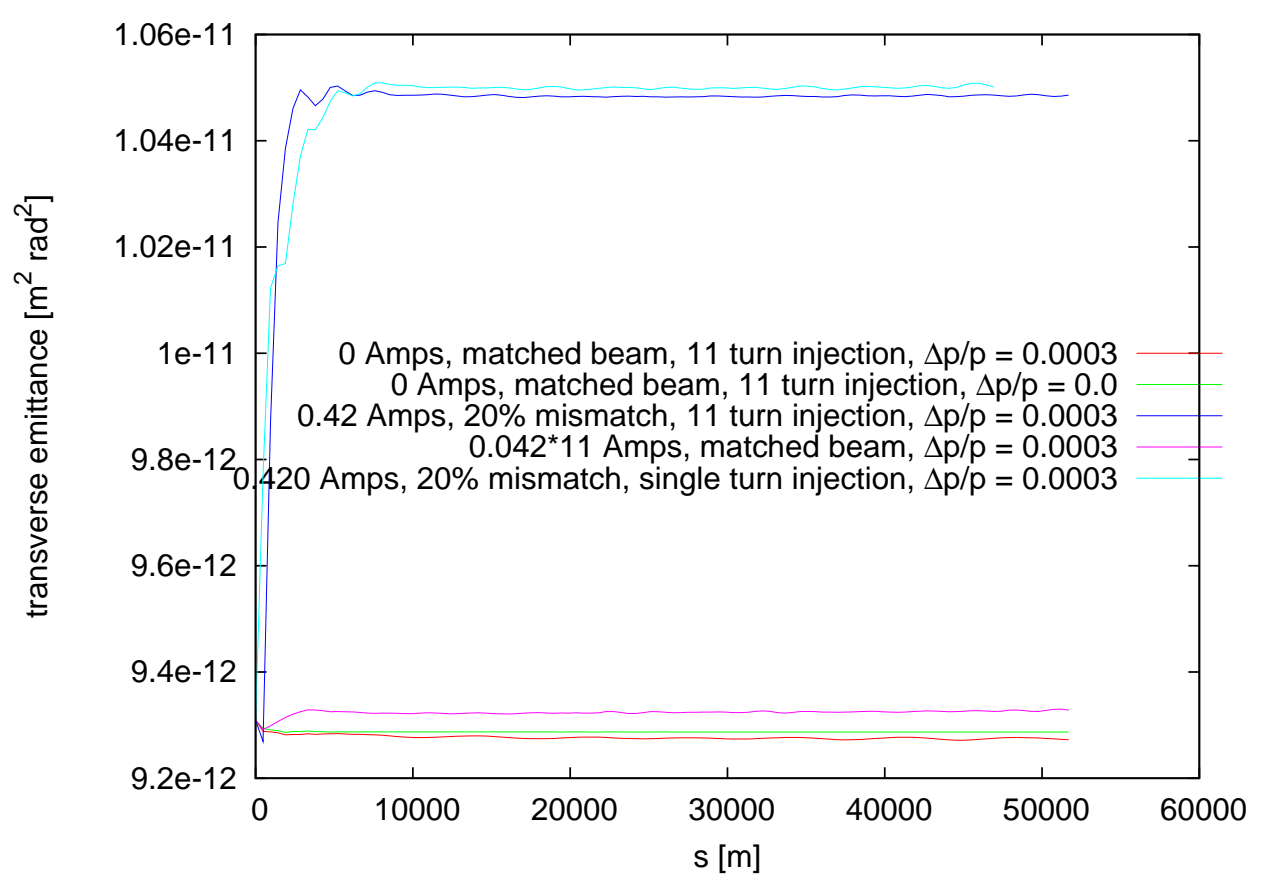

FIG. 16: Normalized 4-D transverse emittance in $\mathrm{m}^{2} \mathrm{rad}^{2}$ for different initial conditions. The red and green curves correspond to a matched beam, with space-charge effects turned off (0 Amps) with and without a momentum spread of 0.0003 , respectively. The purple and light blue curves correspond to a beam of 0.420 Amps total current and momentum spread of 0.0003 , matched and mismatched respectively. Multi-turn injection of 11 turns is used in all of the above cases. The dark blue curve corresponds to a single turn injection simulation of a 0.420 Amp mismatched beam with 0.0003 momentum spread.

\section{CONCLUSIONS}

In this paper we presented Synergia, a package for simulation of linear and circular accelerators with self-consistent treatment of space charge and the ability to use arbitrary order transfer maps for modeling single-particle optics. Synergia provides the tools necessary for non-expert users to easily port and use the package. The user interface takes advantage of the flexibility of Python to provide a complete and highly configurable system. The parallel implementation allows us to perform large scale simulations on modern supercomputers and clusters. We have verified the accuracy of our implementation by comparing Synergia to semi-analytic results and other codes.

In our initial application of Synergia, we studied beam behavior during the first hundred turns after injection in the Fermilab Booster. We calculated the particle tune spread and found that the majority of the particles experience a tune shift much smaller than that predicted by the Laslett formula. We also studied some mechanisms of beam halo creation and emittance growth. We used the kurtosis of the beam distribution as a quantitative measure of halo production. We found that if the injected beam is matched including spacecharge effects there is no apparent emittance growth or halo creation. In the case of a mismatched beam, we found that both chromatic and space-charge effects are important in creating halo and emittance growth. A more detailed study of such effects and their 
dependence on initial conditions will follow in a future paper.

\section{ACKNOWLEDGMENTS}

The authors would like to thank Dr. Leo Michelotti for providing the mxyzptlk/beamline libraries and for many useful discussions regarding single particle optics. For our research we used resources of the Fermilab Lattice QCD group, operated by the Fermilab Computing Division as well as resources of the National Energy Research Scientific Computing Center, which is supported by the Office of Science of the U.S. Department of Energy. This work was performed under the auspices of a Scientific Discovery through Advanced Computing project, "Advanced Computing for $21^{\text {st }}$ Century Accelerator Science and Technology", which is supported by the US DOE/SC Office of High Energy Physics and the Office of Advanced Scientific Computing Research.

[1] F. Jones, G. H. Mackenzie, and H. Schonauer, Particle Accelerators,vol. 31, 199 (1990).

[2] S. Machida, in Computational Accelerator Physics, edited by R. Ryne,AIP Conf. Proc. No. 297 (AIP, New York, 1994), p. 459.

[3] J. Galambos, J. Holmes, D. Olsen, A. Luccio, and J. Beebe-Wang, ORBIT User's Manual, Oak Ridge National Laboratory, SNS/ORNL/AP Technical Note No. 011, 1999.

[4] http://cepa.fnal.gov/psm/aas/Advanced \_Accelerator\_Simulation.html

[5] Booster Staff 1973 Booster Synchrotron ed E L Hubbard Fermi National Accelerator Laboratory Technical Memo TM-405

[6] http://scidac.nersc.gov/accelerator/mli/manual.pdf

[7] J. Qiang, R. D. Ryne, S. Habib and V. Decyk,

[8] L. Michelotti, FERMILAB-CONF-91-159 Presented at 14th IEEE Particle Accelerator Conf., San Francisco, CA, May 6-9, 1991.

L. Michelotti, FERMILAB-FN-535-REV.

L. Michelotti. Published in Conference Proceedings: Automatic Differentiation of Algorithms: Theory, Implementation, and Application. Society for Industrial and Applied Mathematics. First International Workshop on Computational Differentiation. 1991.

L. Michelotti. Published in Conference Proceedings: Advanced Beam Dynamics Workshop on Effects of Errors in Accelerators, their Diagnosis and Correction. Corpus Christi, Texas. October 3-8, 1991. American Institute of Physics: Proceedings No.255. 1992.

[9] F.Christoph Iselin, "The MAD program(Methodical Accelerator Design) Version 8.13/8", Physical Methods Manual, CERN/SL/92, 1992.

[10] The Hierarchical Data Format, http://hdf.ncsa.uiuc.edu/

[11] http://www.opendx.org

[12] Ankenbrandt $\mathrm{C}$ et al 1980 Proceedings of the 11th International Conference on High-Energy Accelerators p 260

[13] Popovic P and Ankenbrandt C 1998 Workshop on Space Charge Physics in High intensity Hadron Rings ed A U Luccio and W T Weng (Woodbury, New York: AIP Conference Proceedings) p 128 
[14] Laslett L.J. 1963 Proceedings of the 1963 Summer Study of Storage Rings, Accelerators and Experimentation at Super-High Energies p. 324

[15] Handbook of Mathematical Functions With Formulas, Graphs, and Mathematical Tables ed M Abramowitz and I A Stegun, National Bureau of Standards Applied Mathematics Series $-55$

[16] T.P. Wangler and K.R. Crandall 2000 Proceedings of the XX International Linac Conference ed A.W. Chao, SLAC Report No. SLAC-R-561, eConf:C000821.

[17] http://lqcd.fnal.gov/

[18] Linux cluster in the beams theory department at Fermilab.

[19] http://www.nersc.gov/alvarez/

[20] http://hpcf.nersc.gov/computers/SP/

[21] $700 \mathrm{MHz}$ Pentium III cluster with Myrinet networking.

[22] Physics of Collective Beam Instabilities in High Energy Accelerators, A W Chao, John Wiley \& Sons, Inc, 1993; F. Sacherer, IEEE Trans. Nucl. Sci. NS-18, 1105 (1971).

[23] The projections of a K-V distribution on the transverse planes are uniform elliptical distributions with sharp boundaries.

[24] There are multiple possible definitions of kurtosis. Abramowitz and Stegun [15] refer to $k$ defined above as the "kurtosis excess."

[25] The 4-D emittance is the square root of the determinant of the covariance matrix of the transverse phase space. 